\title{
Spatially distributed chaos and turbulence in clouds
}

\author{
A. Bershadskii \\ ICAR, P.O. Box 31155, Jerusalem 91000, Israel \\ bershads@gmail.com
}

\begin{abstract}
Spatially distributed chaos (turbulence) in the cumulus and stratiform clouds have been studied using results of direct numerical simulations and radiometric measurements in cloudy atmosphere. It is shown that in the both cases the second order moment of helicity distribution (the LevichTsinober invariant) dominates the kinetic energy spectra.
\end{abstract}

\section{INRODUCTION}

The climate predictions and weather forecasting are heavily dependent on the modelling of the cloud formation under turbulent conditions. Therefore, a vast range of scales should be taken into account - from the cloud microphysical processes to the macroscopic turbulent motions. Both these non-linearly interacting components are poorly understood. The difficulty to conduct measurements of the microphysical properties of the real clouds formation under turbulent conditions (see, for instance, Ref. 1] and references therein) makes the problem formidable. Therefore, the recent direct numerical simulations (see, for instance, Refs. [2], 3], [4] and references therein) trying to take into account the maximum possible variance of the key microscopic and macroscopic processes (collision-coalescence of droplets, condensation-evaporation, the cooling related to the updraft motion inside the cumulus clouds, Reynolds number dependent drag, supersaturation, longwave radiative cooling etc.) in the buoyant turbulent environment are rather important.

In present paper results of the direct numerical simulations have been used in order to develop a comprehensive distributed chaos approach to the hydrodynamic aspects of the problem and are compared to atmospheric measurements.

In the Section II of the paper appearance of the temporally distributed chaos from the deterministic one is studied and compared with results of a direct numerical simulation of the Rayleigh-Bénard thermal convection. In the Section III the spatially distributed chaos in the Rayleigh-Bénard thermal convection has been studied and compared with results of direct numerical simulations. In the Section IV the buoyancy-helical distributed chaos have been introduced, studied and compared with the results of measurements in atmospheric boundary layer during a morning transition from the stable to adiabatic mixed-layer convective conditions. In Section V the distributed chaos has been studied in the cumulus clouds. And, finally, in the Section VI the distributed chaos has been studied in the stratiform clouds and compared with the results of direct numerical simulations and radiometric measurements in cloudy atmosphere

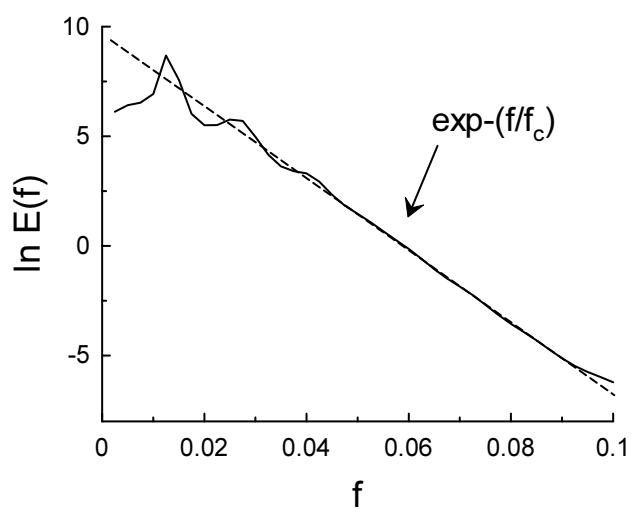

FIG. 1: Logarithm of power spectrum for $z$-component of the Eq. (1) against frequency $f$. The dashed straight line corresponds to Eq. (2).

\section{FROM DETERMINISTIC TO DISTRIBUTED CHAOS}

The deterministic chaos in the thermal convection was discovered by E.N. Lorenz [5] in a simplified model of the Rayleigh-Bénard convection

$$
\frac{d x}{d t}=\sigma(y-x), \quad \frac{d y}{d t}=r x-y-x z, \quad \frac{d z}{d t}=x y-b z
$$

The Rayleigh-Bénard convection is a thermal convection in a layer of fluid, cooled from above and heated from below. The parameters $b=8 / 3, r=28.0, \sigma=10.0$, for instance, provide a chaotic solution. Fig. 1 shows power spectrum of $z$-component (in the semi-logarithmic scales). The spectrum was computed using the maximum entropy method, which provides an optimal resolution for comparatively short data sets [6]. The dashed straight line corresponds to the exponential spectrum

$$
E(f) \propto \exp -\left(f / f_{c}\right)
$$

where $f_{c}=$ const is some characteristic frequency. It is now well known that the exponential spectrum Eq. (2) is a typical spectrum for the deterministic chaos [6]- [10]. 
The Boussinesq approximation for the buoyancydriven flows is (see, for instance, Ref. [11])

$$
\begin{gathered}
\frac{\partial \mathbf{u}}{\partial t}+(\mathbf{u} \cdot \nabla) \mathbf{u}=-\frac{\nabla p}{\rho_{0}}+\sigma g \theta \mathbf{e}_{z}+\nu \nabla^{2} \mathbf{u} \\
\frac{\partial \theta}{\partial t}+(\mathbf{u} \cdot \nabla) \theta=S \frac{\Delta}{H} e_{z} u_{z}+\kappa \nabla^{2} \theta \\
\nabla \cdot \mathbf{u}=\mathbf{0}
\end{gathered}
$$

here $p$ and $\theta$ are the pressure and temperature fluctuation fields $\left(\theta=T-T_{0}(z)\right.$ with $T_{0}(z)$ as a conduction-state profile), $\mathbf{u}$ is the velocity field, $\mathbf{e}_{z}$ is the vertical unit vector, the vertical distance between the two layers is $H$ and the temperature difference between them is $\Delta$ (in the case of the Rayleigh-Bénard convection the $H$ is distance between the bottom and top plates), $\rho_{0}$ is the mean density of the fluid, $\nu$ is the viscosity and $\kappa$ is the thermal diffusivity, the gravity acceleration is $g$ and the thermal expansion coefficient is $\sigma$. For the unstably stratified flows (Rayleigh-Bénard convection) $S=+1$ whereas for the stably stratified flows $S=-1$.

In a direct numerical simulation of the RayleighBénard convection Eqs. (3-5), reported in Ref. [12], dynamics of a global characteristic - normalized heat current through the fluid layer $N(t)$ (the Nusselt number) at constant temperature of the top and bottom surfaces of a cylindrical domain, was studied at the onset of the thermal convection at Prandtl number $\operatorname{Pr}=\nu / \kappa=0.78$ and aspect ratio $\Gamma=4.72$.

Figure 2 shows (in the log-log scales) a broadband windowed power spectrum of the $N(t)$ fluctuations obtained at $\delta=\left(R a-R a_{c}\right) / R a_{c}=0.614$, where $R a_{c}$ is the Rayleigh number at the convective threshold $(R a=$ $\left.\sigma g \Delta H^{3} / \nu \kappa\right)$. The spectral data were taken from Fig. 3 of the Ref. [12]. Observations performed in the Ref. [12] indicate that the spectrum shown in the Fig. 2 is dominated mainly by the nucleation of dislocation pairs.

The dashed curve in the Fig. 2 indicates the exponential spectrum Eq. (2) (position of the characteristic scale $f_{c}$ is shown in the Fig. 2 by a dotted arrow). As it was already mentioned above such spectrum is an usual characteristic of smooth bounded deterministic dynamics.

With increase of the parameter $\delta$ the convection dynamics becomes more and more complicated and at the value $\delta \simeq 3$ time-series of the $N(t)$ fluctuations are already random-like (the dynamics in this case is dominated mainly by the the roll pinch-off events [12]). In order to understand this transition one should take into account that with the increase of $\delta$ the parameter $f_{c}$ in the Eq. (2) becomes fluctuating and one should use an ensemble average over this fluctuating parameter (with

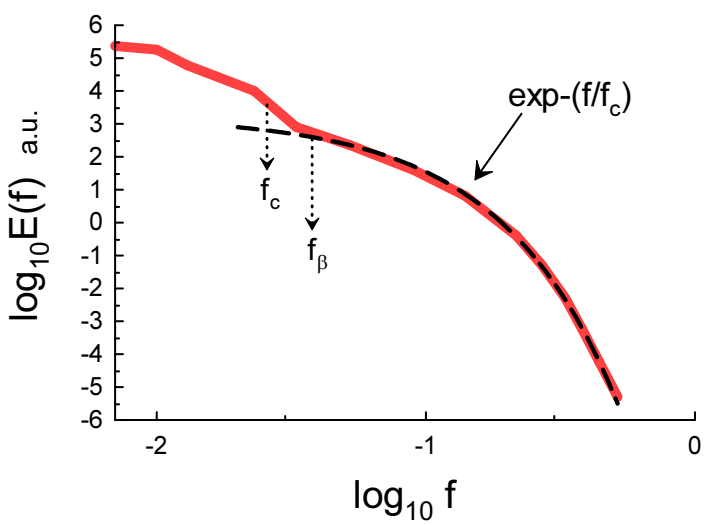

FIG. 2: Windowed power spectrum of the $N(t)$ fluctuations at $\delta=0.614$.

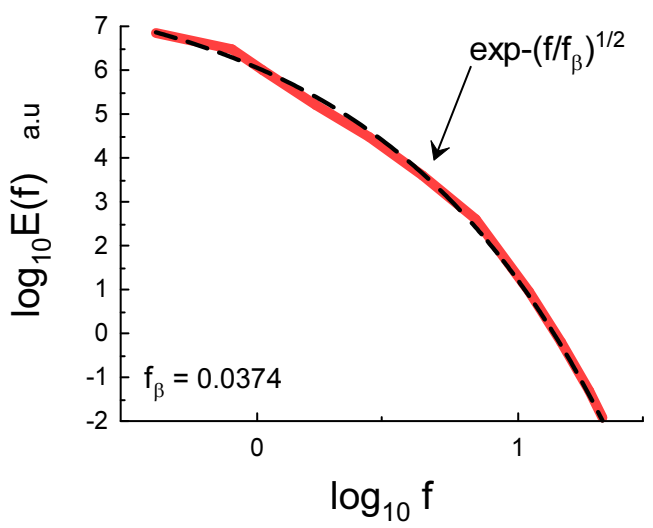

FIG. 3: Windowed power spectrum of the $N(t)$ fluctuations at $\delta=3$.

certain distribution $P\left(f_{c}\right)$ ) to compute the power spectrum

$$
E(f)=\int P\left(f_{c}\right) \exp -\left(f / f_{c}\right) d f_{c}
$$

To find $P\left(f_{c}\right)$ let us recall that despite the thermal convection is not an Hamiltonian system there is a possibility to introduce an effective action $I_{e}$ for such systems in the case of chaotic/stochastic dynamics (see Ref. 13] and references therein). The basic dynamic relationship involving the action is

$$
u_{c} \propto I_{e}^{1 / 2} f_{c}^{1 / 2}
$$

where $u_{c}$ is a characteristic velocity and $I_{e}$ can be considered as an adiabatic invariant [14]. Providing Gaussian (normal) distribution of the characteristic velocity (see, for instance, Ref. [15]) we obtain

$$
P\left(f_{c}\right) \propto f_{c}^{-1 / 2} \exp -\left(f_{c} / 4 f_{\beta}\right)
$$


where $f_{\beta}=$ constant.

Substitution of the Eq. (8) into Eq. (6) results in

$$
E(f) \propto \exp -\left(f / f_{\beta}\right)^{1 / 2}
$$

Figure 3 shows a windowed power spectrum of the $N(t)$ fluctuations obtained at $\delta=3$. The spectral data were taken from Fig. 3 of the Ref. [12]. The dashed curve in the Fig. 3 indicates the stretched exponential spectrum Eq. (9). It follows from comparison of the Figs. 2 and 3 that the stretched exponential power spectrum in the Fig. 3 is tuned to the low-frequency edge of the exponential spectrum shown in Fig. $2-f_{\beta}$ (the second dotted arrow in the Fig. 2). It is natural if the distributed chaos at $\delta=3$ is a result of the development of the deterministic chaos at $\delta=0.614$.

\section{SPATIALLY DISTRIBUTED CHAOS}

The exponential power spectra are typical for deterministic chaos (or for onset of turbulence) not only in the frequency domain but for the wavenumber domain as well (see, for instance Refs. [7],[16] and references therein)

$$
E(k) \propto \exp \left(-k / k_{c}\right)
$$

With transition to turbulence, analogously to the temporal description, fluctuations of the characteristic wavenumber $k_{c}$ can be taken into account by the ensemble averaging

$$
E(k) \propto \int_{0}^{\infty} P\left(k_{c}\right) \exp -\left(k / k_{c}\right) d k_{c} \propto \exp -\left(k / k_{\beta}\right)^{\beta}
$$

where the stretched exponential in the right-hand side of the Eq. (11) is a generalization of the Eq. (9). In this case the probability distribution $P\left(k_{c}\right)$ can be estimated for large $k_{c}$ from the Eq. (11) [17]

$$
P\left(k_{c}\right) \propto k_{c}^{-1+\beta /[2(1-\beta)]} \exp \left(-\gamma k_{c}^{\beta /(1-\beta)}\right)
$$

where $\gamma$ is a constant.

The spatial (wavenumber) version of the relationship Eq. (7) is

$$
u_{c} \propto I_{e} k_{c}
$$

Let us consider a general scaling

$$
u_{c} \propto k_{c}^{\alpha}
$$

If $u_{c}$ has Gaussian distribution (with zero mean) [15] a relationship between $\alpha$ and $\beta$

$$
\beta=\frac{2 \alpha}{1+2 \alpha}
$$

follows immediately from the Eqs. (12) and (14).

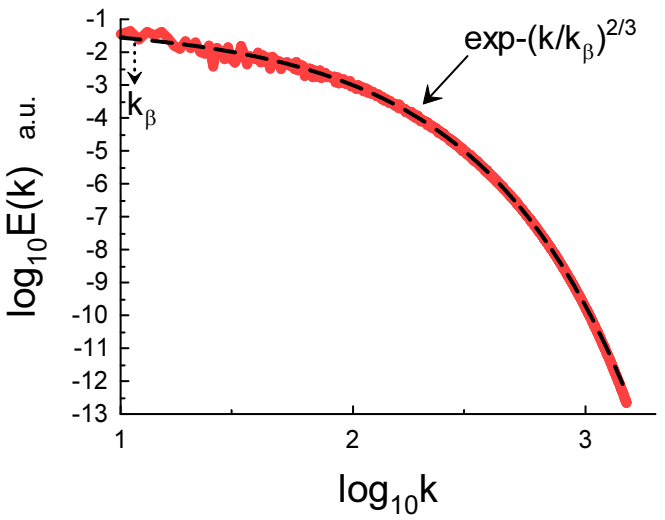

FIG. 4: Spatial kinetic energy spectrum at $\operatorname{Pr}=1$ and $R a=$ $10^{8}$.

Then for the particular case Eq. (13) $\alpha=1$, hence $\beta=2 / 3$, i.e.

$$
E(k) \propto \exp -\left(k / k_{\beta}\right)^{2 / 3} .
$$

In recent Ref. 18 results of a direct numerical simulations of the Rayleigh-Bénard convection at $R a=10^{8}$ (still a moderate value) and the Prandtl number $\operatorname{Pr}=$ $\nu / \kappa=1$ were reported. The free-slip boundary conditions for the velocity field and conducting boundary conditions for the temperature field were used at the top and bottom horizontal plates, whereas at the side walls of the computational domain the periodic boundary conditions were used and random initial condition were applied.

Figure 4 shows spatial kinetic energy spectrum obtained in this DNS. The dashed curve is drawn in order to indicate the stretched exponential spectrum Eq. (16) and the dotted arrow indicates $k_{\beta}$.

\section{HELICAL DISTRIBUTED CHAOS}

Let us consider equation for mean helicity $h=\mathbf{u} \cdot \boldsymbol{\omega}$ (here the vorticity $\boldsymbol{\omega}=\nabla \times \mathbf{u}$ ) corresponding to the Eq. (3) with $\nu=0$

$$
\frac{d\langle h\rangle}{d t}=2 \sigma g e_{z}\left\langle\omega_{z} \theta\right\rangle
$$

where $\langle\ldots\rangle$ denotes an average over volume.

It follows from the Eq. (17) that the helicity generally is not an ideal invariant of the Rayleigh-Bénard convection. However, one can expect that the correlation $\left\langle\omega_{z} \theta\right\rangle$ being considerable at large scales (on the coherent structures) is quickly decreased in the chaotic/turbulent motion when the spatial scales become smaller. Therefore, the second order moment of the helicity distribution (the Levich-Tsinober invariant of the Euler equation [19]) can be still considered as an ideal invariant of the RayleighBénard convection. Indeed, let us divide the volume of 
motion into the cells $V_{j}$ subject to the boundary conditions $\boldsymbol{\omega} \cdot \mathbf{n}=0$ on the bounding surfaces of the cells $-S_{j}$, moving with the fluid [19],[20]. Then for the sells (where the spatial scales are small enough) the helicity, averaged over the cell, can be approximately considered as an ideal invariant. The second order moment can be defined as [20]

$$
I=\lim _{V \rightarrow \infty} \frac{1}{V} \sum_{j} H_{j}^{2}
$$

where

$$
H_{j}=\int_{V_{j}} h(\mathbf{r}, t) d \mathbf{r} .
$$

For a strong convection (e.g. for large $R a$ ) one can expect that the cells with the approximately conserved $H_{j}^{2}$ provide the main contribution to the sum in the Eq. (18) (cf. Ref. [21]) then the second order moment $I$ is approximately an ideal invariant of the Rayleigh-Bénard convection.

For an inertial range of scales the ideal invariant $I$ can be considered as an adiabatic invariant even for the viscous Eq. (3) and, therefore, can replace the $I_{e}$ in the Eq. (13)

$$
u_{c} \propto I^{1 / 4} k_{c}^{1 / 4}
$$

from the dimensional considerations (i.e. $\alpha=1 / 4$ ). Then, it follows from the Eq. (15) that $\beta=1 / 3$, i.e.

$$
E(k) \propto \exp -\left(k / k_{\beta}\right)^{1 / 3}
$$

for the helically dominated convection. Of course, as for the $I_{e}$ dominated convection, this consideration is valid for both the stable and unstable stratification.

Figure 5 shows power spectrum of the longitudinal velocity fluctuations measured at the altitude $100 \mathrm{~m}$ in the atmospheric boundary layer during a morning transition from the stable to adiabatic mixed-layer convective conditions (the spectral data were taken from Fig. 3a of the Ref. [22]). The high Reynolds number data were obtained with a small unmanned aerial system. The dashed curve is drawn in the Fig. 5 in order to indicate the stretched exponential spectrum Eq. (21) and the dotted arrow indicates position of $k_{\beta}$.

\section{DISTRIBUTED CHAOS IN CUMULUS CLOUDS}

In cumulus clouds the buoyancy-driven thermal convection is complicated by collision-coalescence of droplets with hydrodynamic interaction and Reynolds number dependent drag. Nonlinear interaction between turbulent mixing and the condensation-evaporation process

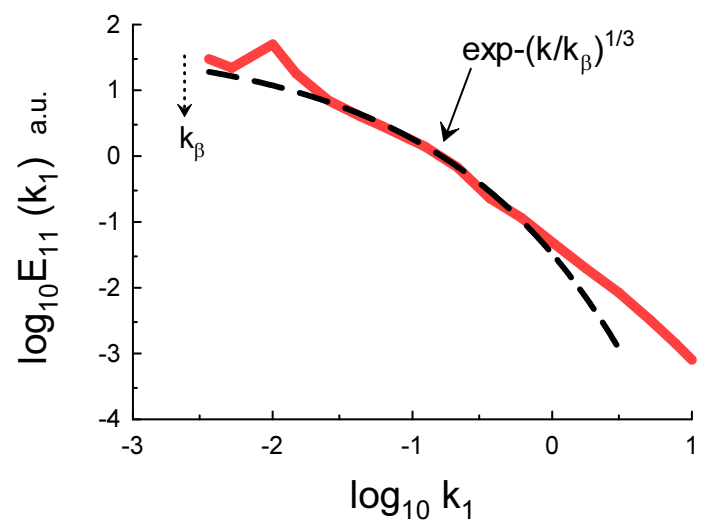

FIG. 5: Power spectrum of the longitudinal velocity fluctuations measured at the altitude $100 \mathrm{~m}$ in the high Reynolds number atmospheric boundary layer during a morning transition from the stable to adiabatic mixed-layer convective conditions.

(in presence of large numbers of droplets) should also strongly affect the convection and evolution of cloud droplets.

There exist many models for description of this complex system. For instance, it was suggested (see recent Ref. [2] and references therein) to consider a small air parcel (a cubic box with volume $L^{3}$ ) ascending inside the core region of a (maritime) cumulus cloud. In the sufficiently small parcel the fluctuating quantities can be considered as statistically homogeneous and the periodic boundary conditions in all three directions can be used. One have to consider corresponding equations describing dynamics of the parcel, flow and droplets.

To describe dynamics of the parcel one can use equations

$$
\frac{\langle d \mathcal{H}(t)\rangle}{d t}=\langle W(t)\rangle \quad \frac{d\langle W(t)\rangle}{d t}=\langle B(t)\rangle
$$

where $B(t)$ is a buoyancy force acting on the parcel, $W(t)$ and $\mathcal{H}(t)$ are corresponding updraft (vertical) velocity and altitude associated with the parcel.

To describe the flow velocity field $\mathbf{u}$, temperature $\theta$ and water vapor mixing ratio $q$ one can use the Boussinesq approximation

$$
\begin{gathered}
\frac{\partial \mathbf{u}}{\partial t}+(\mathbf{u} \cdot \nabla) \mathbf{u}=-\frac{\nabla p}{\rho_{0}}+(B-\langle B(t)\rangle) \mathbf{e}_{z}+\nu \nabla^{2} \mathbf{u}+\mathbf{f} \\
\frac{\partial \theta}{\partial t}+(\mathbf{u} \cdot \nabla) \theta=-\Gamma u_{z}+\frac{L_{v}}{c_{p}}\left(C_{d}-\left\langle C_{d}\right\rangle\right)+\kappa \nabla^{2} \theta \\
\frac{\partial q}{\partial t}+(\mathbf{u} \cdot \nabla) q=-\left(C_{d}-\left\langle C_{d}\right\rangle\right)+\kappa_{q} \nabla^{2} q \\
\nabla \cdot \mathbf{u}=\mathbf{0}
\end{gathered}
$$




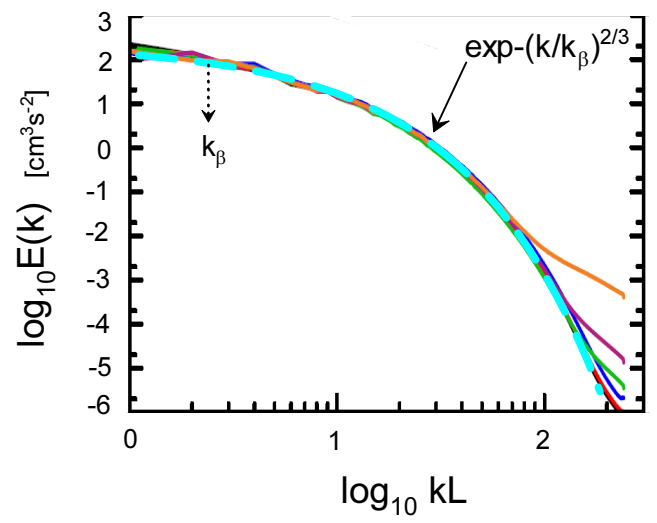

FIG. 6: Kinetic energy spectrum for $R e_{\lambda}=104$.

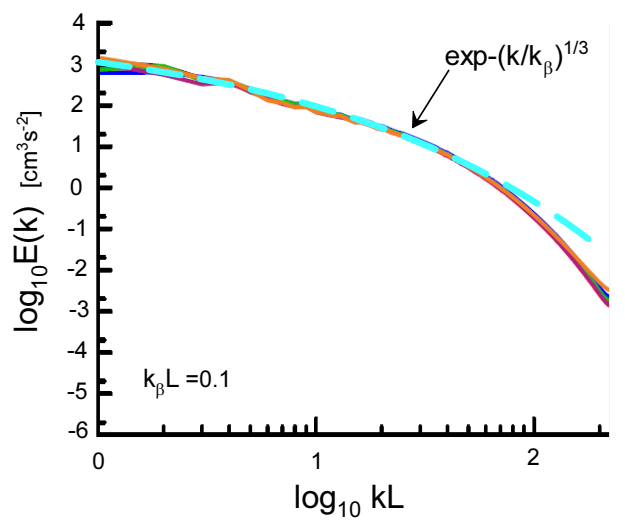

FIG. 7: Kinetic energy spectrum for $R e_{\lambda}=167$.

in the local coordinate system associated with the parcel. In these equations $\mathbf{f}$ is an external (random) force, $\Gamma$ represents the cooling effect related to the ascending motion of the parcel, $C_{d}$ represents latent mass exchange through condensation in the Eq. (26) and heat release in the Eq. (25) (see for more details the Ref. [2]).

For the system Eqs. (24-27) the generalized action $I_{e}$ and second order moment of helicity $I$ are still adiabatic invariants for the inertial range of scales. Let us show this for the second order moment of helicity $I$.

The equation of the mean helicity for the ideal case $(\nu=0)$ is

$$
\begin{gathered}
\frac{d\langle h\rangle}{d t}=2\langle\boldsymbol{\omega} \cdot \mathbf{F}\rangle \\
\mathbf{F}=(B-\langle B\rangle) \mathbf{e}_{z}+\mathbf{f}
\end{gathered}
$$

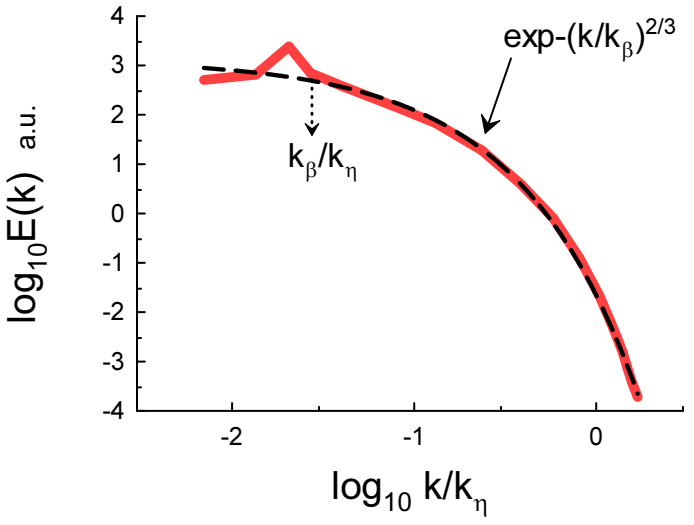

FIG. 8: Time-averaged kinetic energy spectrum for $R e_{\lambda}=$ 130.

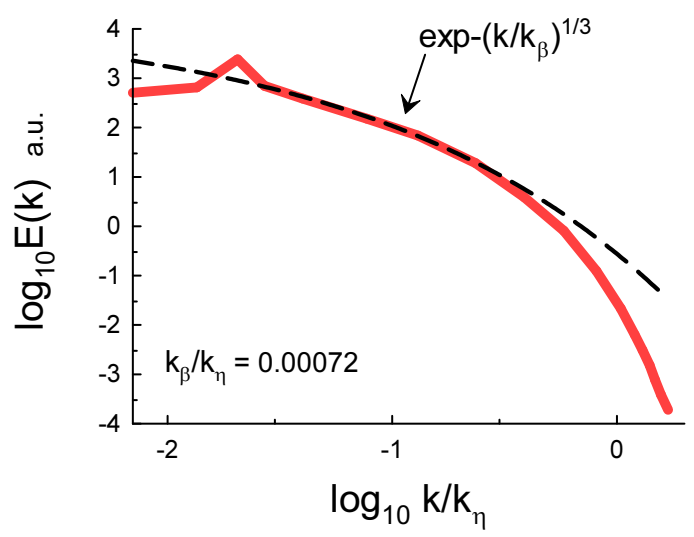

FIG. 9: The same as in the Fig. 8 but with the helically fitted (cf. Eq. (21)) inertial subrange.

(cf. Eq. (17)). If the correlation $\langle\boldsymbol{\omega} \cdot \mathbf{F}\rangle$ is negligible or it is not negligible for the large scales only then the consideration of the Section IV can be readily generalized for the Eq. (28) and the second order moment of helicity $I$ is an adiabatic invariant for the inertial range of scales (in the former case it is an adiabatic invariant for the large scales as well). A direct numerical simulation with the above described model was performed in the Ref. 2] and figure 6 shows the kinetic energy spectrum obtained in this simulation for the Taylor-Reynolds number [15] $R e_{\lambda}=104$ (the specral data were taken from Fig. 7 of the Ref. 2]). Different colors correspond to different times of the system's evolution: from $t=10 \mathrm{~s}$ (black) to $t=600 \mathrm{~s}$ (orange). The dashed curve indicates correspondence to the Eq. (16) (the action invariant $I_{e}$ dominated distributed chaos).

Figure 7 shows the kinetic energy spectrum obtained in this simulation for the Taylor-Reynolds number $R e_{\lambda}=$ 
167 (the specral data were taken from Fig. 7 of the Ref. [2]). Different colors correspond to different times of the system's evolution: from the $t=10 \mathrm{~s}$ (black) to the $t=600 \mathrm{~s}$ (orange). The dashed curve indicates correspondence to the Eq. (21) (the helically dominated distributed chaos).

\section{STRATIFORM CLOUDS}

\section{A. Direct numerical simulations}

In the stratiform clouds the updraft velocity of the parcel is about zero [23], 24]. This results, in particular, in absence of the related cooling effect. Therefore, the condensational growth of the cloud droplets is mainly driven by supersaturation fluctuations (see, for instance, a recent Ref. [3] and references therein).

In the Ref. 3] a direct numerical simulation of a model corresponding to the stratiform clouds was performed with especial attention to the phenomenon of the cloud-droplet growth due to supersaturation fluctuations affected by turbulence (see the Ref. 3] for more detail). Figure 8 shows a time-averaged kinetic energy spectrum obtained in this simulation for $R e_{\lambda}=130$ (the spectral data were taken from Fig. 1a of the Ref. [3]). The wavenumber is normalized by the Kolmogorov wavenumber $k_{\eta}=2 \pi / \eta$, where $\eta=\left(\langle\varepsilon\rangle / \nu^{3}\right)^{-1 / 4}$ is the Kolmogorov (dissipative) scale and $\langle\varepsilon\rangle$ is the mean dissipation rate due to viscosity [15]. The dashed curve indicates correspondence to the Eq. (16) (the action invariant $I_{e}$ dominated distributed chaos).

Figure 9 shows the same spectral data but with the dashed curve indicating the helically dominated distributed chaos Eq. (21). One can see that there already exists an inertial subrange with the helically dominated distributed chaos, and one can expect that this subrange will be increased with $R e_{\lambda}$ (cf. Figs. 6 and 7 ).

In the Ref. 4] results of a direct numerical simulation of stratocumulus cloud-top turbulence were reported (see for more details of the setting and simulations in the Refs. [25], 26]). In the cloud-top region longwave radiative cooling results in convective instability, that is a major source of chaotic (turbulet) motion in the cloud's region.

Figure 10 shows longitudinal spectrum of the horizontal velocity obtained in this simulation (the spectral data were taken from Fig. 1a of the Ref. [3], excluding the data for the upper part of cloud). Figure 11 shows corresponding transverse spectrum and again dashed curve indicates the helically dominated distributed chaos Eq. (21). Figure 12 shows longitudinal spectrum of the horizontal velocity obtained in this simulation for the upper part of cloud. The dashed curves in the Figs. 10-12 indicate the helically dominated distributed chaos Eq. (21).

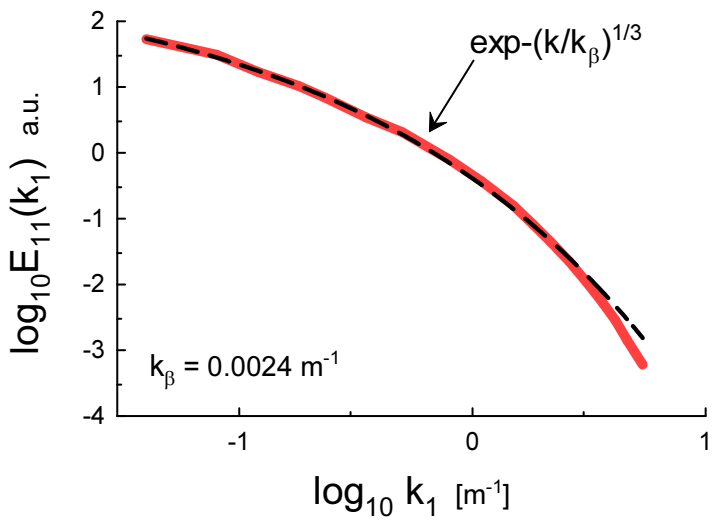

FIG. 10: Longitudinal spectrum of the horizontal velocity.

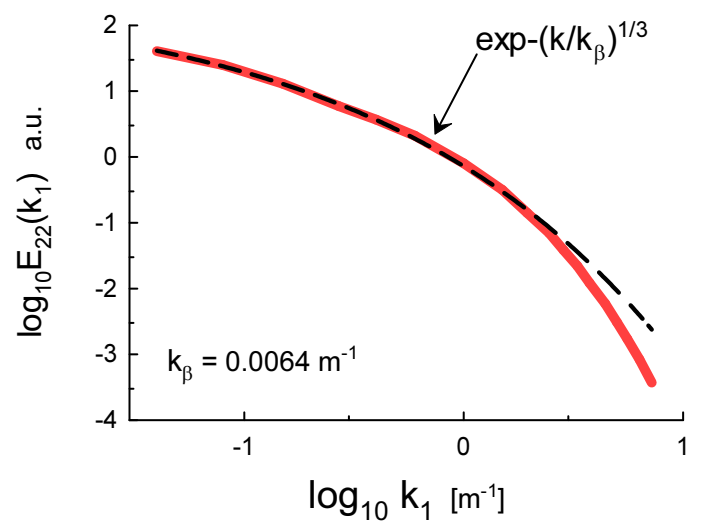

FIG. 11: Transverse spectrum of the horizontal velocity.

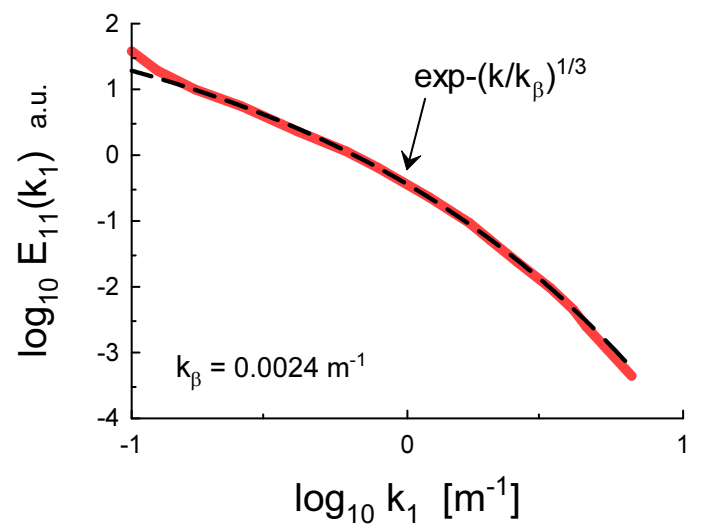

FIG. 12: Longitudinal spectrum of the horizontal velocity for upper part of cloud. 


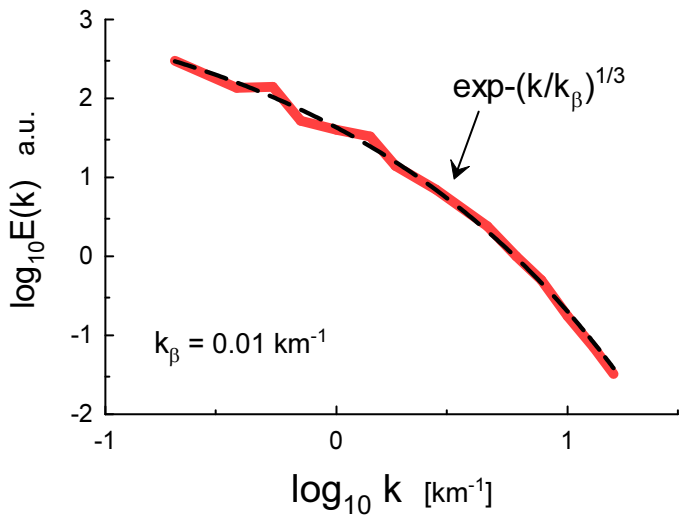

FIG. 13: 1D power spectrum of the radiance in the NorthSouth direction (DF).

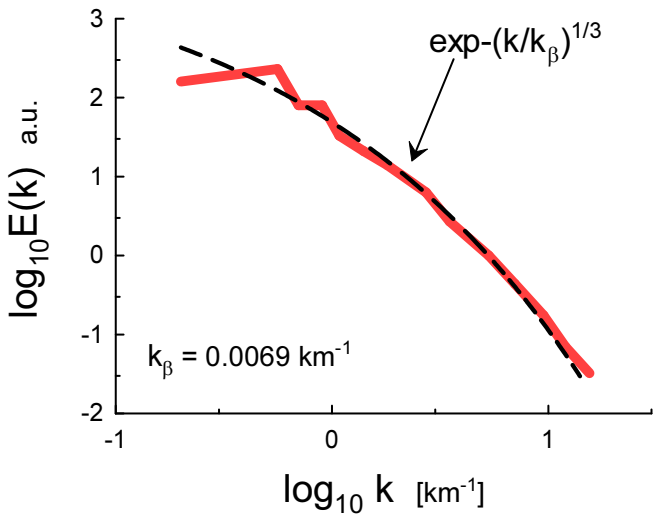

FIG. 14: 1D power spectrum of the radiance in the NorthSouth direction (DA).

\section{B. Radiometric measurements in cloudy atmosphere}

The results of radiometric measurements made by the airborne Multiangle Imaging Spectro-Radiometer (AirMISR), on the NASA ER-2 high-altitude $(\sim 20 \mathrm{~km})$ aircraft, were reported in Ref. 27]. The measurements were made for the blue channel radiance $(\lambda=0.443 \mu \mathrm{m})$ over continental single-layer low stratocumulus clouds. Therefore, one can expect that the radiance fluctuations were produced mainly by the velocity field at the top of the clouds.

Figure 13 shows 1D power spectrum of the radiance in the North-South direction: zenith angle $70.5^{\circ}$ and scattering angle $140.8^{\circ}$ (the spectral data were taken from Fig. 7-DF of the Ref. [27]). Figure 14 shows 1D spectrum of the radiance in the North-South direction: zenith angle $70.5^{\circ}$ and scattering angle $72.3^{\circ}$ (the

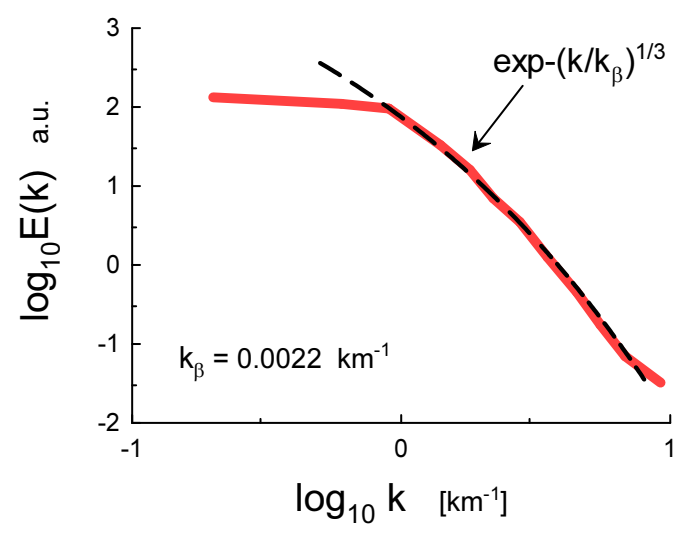

FIG. 15: 1D power spectrum of the radiance in the East-West direction (DF).

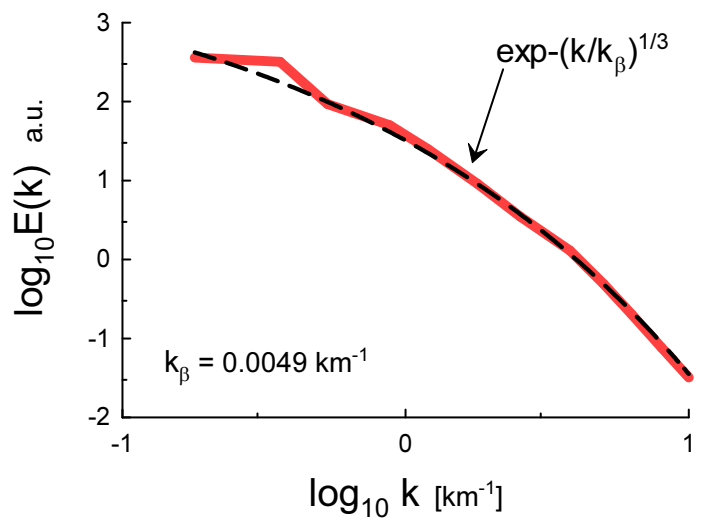

FIG. 16: 1D power spectrum of the radiance in the East-West direction (DA).

spectral data were taken from Fig. 7-DA of the Ref. 27]). The DF and DA camera's view angles are the most oblique ones and for them the cloud gaps close about completely.

Figure 15 shows 1D spectrum of the radiance in the EastWest direction (the spectral data were taken from Fig. 8-DF of the Ref. 27] ). Figure 16 shows $1 D$ spectrum of the radiance in the the East-West direction direction (the spectral data were taken from Fig. 8-DA of the Ref. 27]). The dashed curves in the Figs. 13-16 indicate the helically dominated distributed chaos Eq. (21).

\section{ACKNOWLEDGEMENT}

I thank E. Levich for stimulating discussions, and R. Samuel and M.K. Verma for sharing their data. 
[1] H. Siebert, S. Gerashchenko, A. Gylfason, K, Lehmann, L,R. Collins, R.A. Shaw and Z. Warhaft, Atmos. Res., 97436 (2010).

[2] I. Saito and T. Gotoh, New J. Phys., 20023001 (2018).

[3] X-Y. Li, G. Svensson, A. Brandenburg and N.E.L. Haugen, Atmos. Chem. Phys., 19, 639 (2019).

[4] M. Wacawczyk, E.O. Akinlabi, J.P. Mellado and S.P. Malinowski, Proc. of the "11th International Symposium on Turbulence and Shear Flow Phenomena" (2019). http://www.tsfpconference.org/proceedings/2019/217.pdf

[5] E.N. Lorenz, J. Atmos. Sci., 20, 130 (1963).

[6] N. Ohtomo, K. Tokiwano, Y. Tanaka et. al., J. Phys. Soc. Jpn. 641104 (1995).

[7] J. E. Maggs and G. J. Morales, Phys. Rev. Lett. 107, 185003 (2011); Phys. Rev. E 86, 015401(R) (2012); Plasma Phys. Control. Fusion 54124041 (2012)

[8] D.E. Sigeti, Phys. Rev. E, 52, 2443 (1995).

[9] J. D. Farmer, Physica D, 4, 366 (1982).

[10] U. Frisch and R. Morf, Phys. Rev., 23, 2673 (1981).

[11] A. Kumar, A.G. Chatterjee and M.K. Verma, Phys. Rev. E, 90, 023016 (2014)

[12] M.R. Paul, M.C. Cross, P.F. Fischer, and H.S. Greenside, Phys. Rev. Lett., 87, 154501 (2001).

[13] G.L. Eyink, Physica D, 239, 1236 (2010).

[14] R.Z. Sagdeev, D.A. Usikov, G.M. Zaslavsky, Nonlinear Physics: from the Pendulum to Turbulence and Chaos
(Harwood, New York, 1988)

[15] A. S. Monin, A. M. Yaglom, Statistical Fluid Mechanics, Vol. II: Mechanics of Turbulence (Dover Pub. NY, 2007).

[16] S. Khurshid, D.A. Donzis, and K.R. Sreenivasan, Phys. Rev. Fluids, 3, 082601(R) (2018).

[17] D.C. Johnston, Phys. Rev. B, 74, 184430 (2006).

[18] S. Vashishtha, M.K. Verma and R. Samuel, Phys. Rev. E., 98, 043109 (2018).

[19] E. Levich and A. Tsinober, Phys. Lett. A 93, 293 (1983).

[20] H.K. Moffatt and A. Tsinober, Annu. Rev. Fluid Mech., 24, 281 (1992)

[21] A. Bershadskii and A. Tsinober, Phys. Rev. E, 48, 282 (1993).

[22] C.A. Canter and S.C.C. Bailey, Proc. of the "11th International Symposium on Turbulence and Shear Flow Phenomena" (2019). http://www.tsfpconference.org/proceedings/2019/304.pdf

[23] J.G. Hudson and G. Svensson, J. Appl. Meteorol., 34, 2655 (1995).

[24] A.V. Korolev, J. Atmos. Sci., 52, 3620 (1995).

[25] J.P. Mellado, Annu. Rev. Fluid Mech., 49, 145 (2017).

[26] B. Schulz and J.P. Mellado, J. Atmos. Sci., 75, 3245 (2018).

[27] M. Ovtchinnikov and R.T. Marchand, Remote Sensing of Environment, 107, 185 (2007). 